\title{
Neurological Lesions in Leprosy
}

\author{
C. L. CRAWFORD, м.в., CH.B. (N.Z.), M.R.C.P., D.T.м. \& H. \\ Medical Officer (Leprosy), Nigerian Government
}

In the course of a 14-month period in Northern Nigeria an opportunity was presented of examining the variety of neurological lesions in patients with leprosy.

Monrad-Krohn (1923) in a study of 63 leprosy patients concluded that the neurological lesion in leprosy was characteristic of a polyneuritis involving both sensory and motor functions. The involvement was thus distal with the sensory loss being 'glove and stocking' in distribution. Furthermore, only superficial sensory modalities were affected; deep pressure, pressure pain and joint sense not being involved. Kinnier Wilson (1954) confirmed these findings but found some impairment of vibration sense in 3 of his own patients. Cochrane (1964) mentions the occurrence of 'glove and stocking' anaesthesia, but only in primary neuritic leprosy, i.e., leprosy showing only neutral signs with no visible cutaneous lesions or scars of previous cutaneous lesions.

Both Monrad-Krohn and Kinnier Wilson describe the early and isolated involvement of individual nerves such as the ulnar and peroneal nerve in leprosy patients. It is this mononeuritis or mononeuritis multiplex which is regarded by Brand (1964) as causing the motor paralysis and anaesthesia in leprosy. The nerves affected are the ulnar, median, common peroneal, posterior tibial, facial and occasionally the radial. Brand also regards the sensory paralysis as following the same pattern, but in addition to the nerves already mentioned there is a paralysis of the long cutaneous nerves that run down from the upper arm to supply the forearm and hand, and those that run down from the thigh to supply the leg below the knee. The cause of the paralysis and sensory loss is due to oedema and a cellular infiltration in certain sites along the nerve. This leads, firstly to ischaemia and, if prolonged, to destruction of the nerve. Why certain nerves only are affected and special sites along these nerves are involved is not clear, but Brand suggests it is primarily the nearness of the nerve to the skin where the temperature is lower.

\section{MATERIALS AND METHODS}

All types of leprosy were seen, ranging from patients with single skin lesions to those with severe deformities. Twenty patients with neurological lesions were examined in detail. These were predominantly of Hausa origin and were all patients of the Zaria Leprosy Settlement.

The diagnosis of leprosy was made mainly on clinical grounds. In lepromatous patients, infiltration of the ears, absence of eyebrows, depression of the nose, infiltration of the skin and nodulation were all regarded as diagnostic criteria. In 5 of the 9 patients the diagnosis was confirmed bacteriologically. In one patient a skin biopsy showed lepromatous features, and the remaining 3 had been discharged, cured, and were no longer in chemotherapy.

In non-lepromatous patients the diagnosis was made by the character of the skin lesions. In those patients where there were no skin lesions, the diagnosis was made by the appearance of the extremities with loss of digits, ulceration of the feet and contractures. In one patient the diagnosis was confirmed histologically.

There was no clinical evidence of syphilis or vitamin deficiency, and the general state of nutrition was good.

Sixteen patients were male and 4 were female. The age of patients was difficult to obtain but in the males varied from school children to middle age, while the 4 female patients were all young women.

All but one patient had taken dapsone at some time, but only 6 are presently continuing

Neurological Lesions in Leprosy 
this treatment. Three patients are being treated with thiambutosine (DPT).

\section{RESULTS}

These are divided into 3 groups according to the neurological lesion.

\section{Group 1}

Purely sensory polyneuritis: 12 patients.

Eleven of the patients showed loss of sensation to light touch, pinprick, hot and cold, while sensations of deep pressure, pressure pain, vibration and joint sense were fully preserved. The twelfth patient showed preservation to pinprick as well. The distribution of the sensory loss was in a 'glove and stocking' fashion, but the extent varied considerably. In some patients the sensory loss extended from the extremities to the knees and elbows, while in others it extended only to the ankles and wrists. In 3 of the patients the legs only were involved, the fingers and arms being normal. The distribution was thus predominantly distal. There was no motor loss.

Nine of the patients were classified as lepromatous. Of the 3 non-lepromatous patients, one was a long standing patient with no skin lesions, another one had widespread macular lesions over trunk and limbs. The remaining patient, together with one of the lepromatous patients, are described in detail as they illustrate the mode of onset of sensory loss.

Patient $\mathrm{l}-\mathrm{A}$ boy of about 16 years was admitted on 7.1.67 with pain and oedema of both hands, feet and face. There were widespread, moderately well defined, macular lesions over trunk and limbs. Neurological examination was normal. Dexamethasone was started on 7.1.67 to reduce the pain and oedema, starting with 12 tablets of $0.5 \mathrm{mg}$ daily and reducing the dose by $0.5 \mathrm{mg}$ a day, so that the course finished on 18.1.67. This gave relief of pain and oedema, but early in February there was a recurrence of symptoms so that Dexamethasone was recommenced on 10.2.67 and stopped on 17.2.67. This again gave immediate temporary relief, but as soon as the course finished there was another recurrence. A further course of steroids was thought inadvisable and hence Camoquin was started on 13.3.67 and continued until 1.4.67. The pain and oedema finally subsided early in April. Some weeks later it was observed that there were blisters on one of the fingers, and neurological examination then showed sensory loss, involving both hands and feet, of 'glove and stocking' distribution. After 3 months' observation there has been no extension of the sensory loss. A biopsy of a macule on the back showed the following:-

'Slight cellular infiltrate is seen along a few neurovascular pathways in the deep and middle dermis. The infiltrating cells are histiocytes with a relatively large number of lymphocytes and a few plasma cells. Infiltrate is accompanying nerve tissue but there is no obvious intraneural infiltration. A few acid-fast bacilli are to be found mainly in neural tissue. This comes in the lepromatous half of spectrum but there are some slight dimorphous features.'

Patient 2-A young woman was admitted on 6.5.67 complaining of severe pain of the hands and feet. There was marked oedema in this area, but there was no sensory or motor loss. There was a well defined hypopigmented skin lesion in the region of the right elbow and a similar lesion of the lef $t$ upper back. She was treated with Prednisolone $60 \mathrm{mg}$ a day, starting on 9.5.67 and reducing by $5 \mathrm{mg}$ a day, stopping on 20.5.67. This relieved the symptoms, but on 1.6.67 there was a recurrence and the next day she complained of numbness of the hands and feet. Examination showed loss of sensation to light touch, pinprick and some impairment of hot and cold in the hands and feet of 'glove and stocking' distribution. Vibration sense, joint sense, pressure pain and deep pressure were all preserved and there was no motor loss. Prednisolone $60 \mathrm{mg}$ daily was recommenced and the next day there was some return of the sensation of light touch and pinprick in a patchy distribution over the hands and feet. However, it was felt necessary because of the danger of adrenal suppression to reduce the dose of Prednisolone and with this reduction the sensation of light touch became impaired, but pinprick remained in a patchy fashion.

Further observation has been limited to 6 weeks, but there has been no extension of sensory loss.

A biopsy of the skin lesion in the region of the right elbow shows the following:

'There are moderate accumulations of infiltrating cells arranged along neurovascular bundles in the deep and middle dermis. The infiltrating cells are histiocytes with relatively large numbers of lymphocytes and plasma cells. Some of the histiocytes show some epithelioid cell change and these cells are arranged in fairly large accumulations. There is cytoplasmic fusion among them and in a few places there are giant cell formations. Nerves are well surrounded by infiltrate and some show definite intraneural infiltration and partial destruction. No acidfast bacilli we re seen in the sections examined.

'This comes in the tuberculoid half of the leprosy spectrum but the degree of resistance is not particularly high and it can beclassified as dimorphous tuberculoid at present.'

A skin biopsy was taken from the area of anaesthesia on the dorsum

as follows:

'Fairly compact accumulations of infiltrating cells are seen along some neurovascular pathways in the subcutaneous tissue and deep and middle dermis.

\section{Leprosy Review}


In the upper dermis it is a little more widespread. In places it extends up close to the basal layer, but there is no penetration into the epidermis. The infiltrating cells are histiocytes with a relatively large number of lymphocytes and a few plasma cells. Scme of the histiocytes show some epithelioid cell change and there is a tendency for these cells to be clustered together in fairly large groupings. There are a few small giant cell formations among these cells. Nerves show perineural infiltrate and some of them show partial intraneural infiltration. No acid-fast bacill i were seen. Sweat glands appeared normal.'

\section{Group 2}

Sensory polyneuritis, plus mononeuritis:

7 patients.

In 4 patients there was a motor paralysis of the ulnar, median and common peroneal nerves and, in addition, there was sensory loss of 'glove and stocking' distribution, but with preservation of joint sense, vibration sense, deep pressure and pressure pain, as in Group 1 patients.

In 3 patients there was motor paralysis of similar nerves, but there was, in addition, some impairment of joint sense, pressure pain and deep pressure, with sensory loss as before.

The extent of the sensory loss was much greater than in Group 1 patients, involving all 4 limbs in each patient and, in most patients extending from the extremities to above the knee and elbow.

All these patients were of long duration and were all inactive clinically. None were lepromatous. One patient had visible, multiple, welldefined macules, mainly on the limbs; one patient had atrophic macules on the trunk; the remainder had no visible lesions. The majority of patients had contractures and some had loss of fingers and toes, with ulcers on the soles of the feet.

\section{Group 3}

Mononeuritis: one patient.

This patient had involvement of the right ulnar nerve, with thickening at the elbow. There was slight but definite motor involvement of the right ulnar nerve with weakness of abduction of the fingers. There was loss of light touch, pinprick and hot and cold in the little finger and half the ring finger, but there was no loss of joint and vibration sense.

\section{A UTONOMIC INVOLVEMENT}

In all the 3 groups of patients there was no postural hypotension as judged by blood pressure readings in prone and standing positions, and none of the patients admitted to nocturnal or post-prandial diarrhoea. No observations were made on pilomotor function as Northern Nigerian patients have very little body hair. In patients with sensory polyneuritis there was extensive loss of sweating which corresponded generally to the areas of loss of sensation.

\section{DISCUSSION}

The findings suggest that there are 2 basic types of neurological lesion in leprosy. Firstly, a sensory polyneuritis and, secondly, a mononeuritis or mononeuritis multiplex. The 2 may co-exist or be present separately. Further, the sensory polyneuritis affects mainly superficial modalities. In all but 3 of the patients described deep modalities of sensation were preserved, and this is consistent with the findings of Monrad-Krohn. However, the motor involvement which Monrad-Krohn describes is more characteristic of a mononeuritis rather than part of a polyneuritis"as it does not affect the distal muscles equally, e.g., in the hand the muscle groups affected are consistent with an ulnar or median nerve palsy. The sensory polyneuritis would seem to form the neurological basis for the consequent destruction of fingers and toes, and ulceration of the feet, involving as it does all the digits and soles of the feet. On the other hand the mononeuritis is responsible for the motor paralysis.

Further investigation is needed into autonomic abnormality in leprosy. Though there is sudomotor disfunction, there is no evidence of vasomotor abnormality as judged by symptons and signs of postural hypotension or diarrhoea. Nor have there been any reports of hyperthermia in leprosy patients. With the hot climate and extensive areas of sweat loss, such a finding might be expected if there was any interference with temperature regulating mechanisms. It is possible that only the nerve fibres supplying the sweat glands are affected. 
Purely sensory polyneuritis is not a very common condition. It may be an initial or prominent feature of types of polyneuritis such as alcoholic, diptheritic or arsenical, but invariably there is some motor component. A purely sensory neuropathy has been described in association with carcinoma of the lung (Brain, 1955) but in contrast to the lesion of leprosy there was gross impairment of posture with loss of tendon reflexes.

The polyneuritic element of sub-acute combined degeneration of the cord is characterised by loss of superficial sensory modalities of 'glove and stocking' type distribution which is similar to leprosy, but there is usually some motor element and the clinical signs are influenced by the posterior and pyramidal tract involvement.

Diabetic polyneuropathy is predominantly sensory with anaesthesia of lower limbs which may be patchy or complete. Paresis may be absent despite profound sensory loss. Although loss of vibration sense in the lower limbs is almost constant in adults with diabetes, vibration sense may be preserved in patients with severe diabetic neuropathy. The loss is gradual, and it rarely recovers. For these reasons Simpson (1962) suggests that loss of vibration sense should be classified separately. If this is so then there is some resemblance to the lesion of leprosy. However, autonomic disfunction is a prominent feature of diabetic polyneuropathy. Sweating, vasomotor and pilomotor control are defective, leading to dependent oedema softening of the skin and hypotension. With the exception of loss of sweating these are not obvious features in leprosy patients.

There are several familial and congenital sensory neuropathies which are of interest in their relation to leprosy. Thus, familial dysautomnia is associated with indifference to pain, but, in addition, there is disordered automnic function and, moreover, there is increased rather than lack of sweating.

Hereditary sensory radicular neuropathy is a progressive disease, which involves the extremities, but all sensory modalities are affected and the tendon reflexes are impaired or lost.
Congenital insensitivity to pain with anhydrosis (Swanson, 196:3) has been described in 2 male children who sustained mutilating injuries of bones and joints since infancy without showing any painful reaction. This insensitivity to pain was accompanied by a complete inability to sweat, and it is of interest that, apart from an abnormal cold pressor test, vasomotor function was normal. However, in contrast to leprosy, both superficial and pressure pain were affected and touch sensation was normal. The children were also intolerant of extremes of temperature. A congenital defect of peripheral innervation is postulated as the course of this syndrome. One of the children died and a post-mortem disclosed absence of Lissauer's tract and small dorsal root axons

The clinical findings of sensory polyneuritis of leprosy suggests a very superficial and peripheral neurological lesion of the limbs. This localisation needs confirmation by physiological and pathological studies.

The 2 reports of patients illustrate the abrupt onset of sensory loss. The clinical picture of acute pain and oedema has been described previously (Davison, 1961) and undoubtedly comes under the category of 'reaction'. This has been defined as: 'More or less sudden tissue responses resulting from the liberation of bacilli or their products into the tissues the manifestations of which can either be local or systemic.' (Cochrane, 1964).

Cochrane has strongly emphasised the acute onset and widespread paralysis in reactional dimorphous states associated with gross oedema of face and limbs, fever, tenderness and ulceration of the skin lesions and severe involvement of the nerves. What has not apparently been so well emphasised is that the oedema may be the major manifestation, and it is noteworthy in the 2 patients described that there was little alteration in the skin lesions or pain in the peripheral nerves. Furthermore, the oedema is associated with sensory loss of the extremities of 'glove and stocking' type distribution, and the process is occurring simultaneously in all 4 limbs. 
The proportion of patients with sensory polyneuritis arising in this way, compared with a more insidious onset, can only be assessed after a larger number of patients are examined.

\section{SUMMARY}

The clinical features of a purely sensory form of polyneuritis in leprosy patients are described. These include a selective involvement of superficial sensory modalities with anhydrosis but without other autonomic involvement. This polyneuritis may occur separately or be associated with the more well-known mononeuritis or mononeuritis multiplex. Other types of sensory polyneuritis and congenital sensory neuropathies are compared and a peripheral and superficial neurological localisation is postulated.

The abrupt onset of sensory loss and the simultaneous involvement of all 4 limbs is illustrated by 2 case history reports.
ACKNOWLEDGEMENT

I wish to thank Dr. D. J. Harman of the Leprosy Study Centre, London, for the biopsy reports.

\section{REFERENCES}

BRAIN, R. (1955). Diseases of the Nervous System. Oxford University Press. P. 747.

BRAND, P. W. (1964). Leprosy in Theory and Practice (Ed. Cochrane, R. G.). Bristol. John Wright. Pt. 447. Cochrane, R. G. (1964). Ibid. Pp. 274, 332, 334.

Davison, A. R. (1961). Acute Oedema of Hands and Feet in Leprosy. Int. .J. Lepr., Washington, 1961, Jan.-Mar., 29, 1, 29-33.

KINNIER WILSON (1954). Neurology. 2nd Edition (Ed. Ninian Bruce). Butterworth. P. 748-755.

Monrad-Krohn, G. H. (1923). The Neurological Aspect of Leprosy. Christiania. Dybwad.

Simpson, John A. (1962). Modern Trends in Neurology. Butterworth (Ed. Denis Williams). P. 274.

Swanson, A. G. (1963). Congenital Sensitivity to Pain with Anhydrosis. Arch. Neurol., 8, 1963, p. 299. 